\title{
OPTIMIZING THROUGHPUT OF A MULTI-ROOM PROTON THERAPY TREATMENT CENTER VIA SIMULATION
}

\author{
Stuart Price \\ Bruce Golden \\ Robert H. Smith School of Business \\ University of Maryland \\ College Park, MD 20742, USA
}

Edward Wasil

Kogod School of Business

American University

Washington, DC 20016, USA

\author{
Hao Howard Zhang \\ University of Maryland School of Medicine \\ Baltimore, MD 21201, USA
}

\begin{abstract}
More than half of all cancer patients in the United States receive radiation therapy during the course of their treatment. The main goal of radiation therapy is to optimize the trade-off between delivering a high and conformal dose to the target and limiting the doses to critical structures. The rationale for using proton beams instead of photon beams is the feasibility of delivering higher doses to the tumor while maintaining the total dose to critical structures or maintaining the target dose while reducing the total dose to critical structures. Despite its promise as a treatment, adoption of proton therapy (PT) is limited by the high cost of building the required facilities. New facilities should be built with layouts that are designed to treat more patients to recoup the initial investment. We examine several facility layouts and scheduling plans to minimize idle equipment and maximize total patient throughput.
\end{abstract}

\section{INTRODUCTION}

The Maryland Proton Treatment Center involves an investment of more than \$200 million to build a proton therapy treatment center that includes imaging equipment, offices, and accommodations for patients (Roylance 2010). This large initial investment is what makes proton therapy so expensive for patients. Due to the large initial investment, only 10 proton therapy treatment centers are operating in the United States currently (there are more than 10,000 linear accelerators used for x-ray intensity modulated radiation therapy (IMRT) worldwide) with eight more in development (National Association for Proton Therapy 2013). A cyclotron is needed to accelerate protons to the speed required for treatment. It is a much larger piece of equipment than the linear accelerators used for photons in x-ray IMRT. Typically, a new facility is built to house the equipment needed for PT, whereas linear accelerators can be installed in existing hospitals. When building an entirely new facility, there are few limitations that are imposed on placing the equipment. A wide array of layouts and configurations of the equipment can be considered. Our goal is to optimize the layout for patient throughput, while maintaining reasonable patient wait times. First, we need to determine how many gantry and imaging rooms are needed to ensure the cyclotron is 
used at full capacity. Second, we need to determine if the imaging rooms are fully connected to the gantry rooms or are there dedicated imaging rooms. Third, given a layout that can utilize the cyclotron at or near capacity, we need to determine whether or not the patient arrival rate affects patient wait time and machine idle time. Finally, we need to examine the effect of patient tardiness and absenteeism on patient wait time and machine idle time.

Patients undergoing proton therapy typically receive treatment five days a week for four to five weeks. Daily treatments take less than an hour and do not typically require anesthesia or other drugs that might affect a patient's daily routine. Some patients will come from out of town for treatment and will have an extended stay. The small number of side effects allows for local patients to continue working while receiving treatment. Tardiness of outpatients has been observed in other settings (Alexopoulos et al. 2008) and is likely to occur among local patients.

In Figure 1, we show the flow of patients through the treatment center. Patients enter the center and proceed to a waiting room where they wait for an available imaging room to begin treatment. Each daily treatment begins with a detailed imaging process to ensure the radiation is delivered to the precise treatment location. The biggest advantage of PT over IMRT is its ability to deliver a precise, highly concentrated dose of radiation. When accurately delivered, this results in less damage to the surrounding tissue and fewer side effects (Lundkvist et al. 2005). Accurate delivery is important because the higher radiation levels delivered by PT will easily destroy healthy tissue if accidentally irradiated (Schulz-Ertner and Tsujii 2007). Errors in imaging and patient immobilization have been cited as the cause of adverse reactions in the treatment of prostate cancer (Wroe, Rosenfeld, and Schulte 2007). In remote positioning, the patient is positioned in the imaging room and moves between rooms immobilized on a transporter. Upon entering the imaging room, the patient rests on the transporter and is immobilized and prepared for imaging. A computer tomography (CT) scanner is used to identify the planned treatment volume. Marks are made on the patient's skin that will later help align the proton beam. The transporter with the patient is undocked from the CT scanner and moved to the gantry room. The transporter is docked with the gantry arm and aligned in preparation for treatment. The patient receives radiation from one, two, or three different beam angles. For each beam angle, the radiation is delivered over the cross section of the planned treatment volume from that angle and divided into pixels. Each pixel receives a different dose of radiation. After the patient has been treated, technicians dismount the patient from the transporter and return the transporter to the imaging room. The patient is discharged.
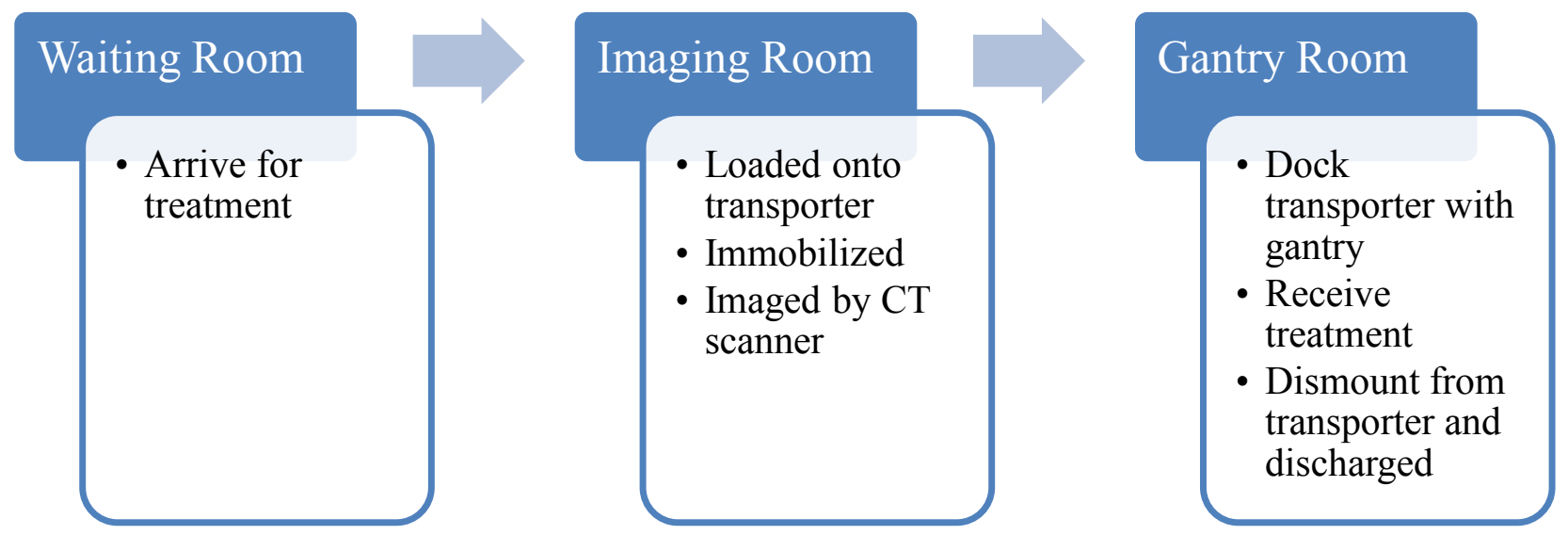

Figure 1: Patient flow through a PT treatment center. Patients may have to wait between rooms.

There is very little published research on the scheduling and operations of PT treatment centers. The Paul Scherrer Institute (PSI) in Switzerland was the first to use remote patient positioning for proton therapy. The process used by PSI is described in the paper by Bolsi et al. (2008). The authors showed that 
remote positioning provided fast patient transport and maintained high accuracy in the delivery of radiation. The benefits of remote patient positioning when compared to traditional in-room positioning in PT treatment centers was examined in a follow-up paper based on simulations using data gathered from PSI (Fava et al. 2012). The authors ran simulations to compare patient throughput of remote and in-room positioning with a variety of transport speeds, imaging times, and beam delivery times. The authors imposed a strict three-minute waiting time limit for patients, so that a limited range of gantry and imaging room configurations were considered.

In this paper, we compare the performance of dedicating an imaging room to each gantry room to fully connecting imaging rooms to gantry rooms. We examine the relationship of patient inter-arrival rate (total daily throughput) to patient wait time and machine idle time.

\section{SIMULATION MODEL}

We built our model using NetLogo 5.0.3, a specialized agent-based simulation language (Wilensky 1999). We chose NetLogo because it has all the capabilities that we require for this project and it is available to the public at no charge. In addition, using an agent-based modeling language, such as NetLogo, will allow the simulation to be extended to include more complex patient, facility, and personnel interactions in future versions of our model. Currently only patient and facility (i.e., the cyclotron) agents are implemented. Our model is run in discrete time with each tick representing five seconds. The treatment times that are used in our model come from an analysis of a PT treatment center using remote positioning due to Fava et al. (2012). The treatment steps and times are shown in Table 1. Treatment times for each step are drawn from a symmetric triangular distribution and are rounded to the nearest tick. A symmetric triangular distribution is used to ensure that all times are bounded and positive. A symmetric triangular distribution provides a clear peak and a wide range of values.

Table 1: Mean time and standard deviation of the steps used in our model based on treatment sessions at the Paul Scherrer Institute (taken from Fava et al. (2012)). Step 2 occurs in the CT scanning room while steps 4 through 10 take place in the gantry room. Beam delivery time based on manufacturer estimate.

\begin{tabular}{lcc}
\hline Treatment Steps & $\begin{array}{c}\text { Mean Time } \\
\text { (minutes) }\end{array}$ & $\begin{array}{c}\text { Range of Values } \\
{[\text { min, max }]}\end{array}$ \\
\hline (1) Patient enters the facility & 0.00 & {$[0.00]$} \\
(2) Imaging & 16.83 & {$[8.50,25.17]$} \\
(3) Move to gantry room & 1.33 & {$[1.33]$} \\
(4) Prepare for beam angles & 6.67 & {$[4.25,9.08]$} \\
(5) First beam angle & 1.25 & {$[1.25]$} \\
(6) Move gantry arm & 1.50 & {$[1.50]$} \\
(7) Second beam angle & 1.25 & {$[1.25]$} \\
(8) Move gantry arm & 1.50 & {$[1.50]$} \\
(9) Third beam angle & 1.25 & {$[1.25]$} \\
(10) Discharge patient and reset gantry room & 4.83 & {$[2.83,6.83]$} \\
\hline
\end{tabular}

In our simulation runs, a patient is randomly assigned a treatment plan with one, two, or three beam angles. For each patient, we record the amount of time waiting for entry into the imaging room and gantry room, and the amount of time waiting in the gantry room for the cyclotron.

The waiting room is where a patient enters the system at the scheduled arrival time. If all imaging rooms are in use, then a count is recorded of the time a patient spends in the waiting room. Otherwise, the patient passes immediately into an available imaging room. For experiments that include a distribution of 
arrival times about a patient's scheduled appointment time, the wait time only includes time spent in the waiting room after the time of the scheduled appointment.

Each imaging room can service only one patient at a time. The patient undergoes a series of procedures in the imaging room. These procedures are the same for each patient and each room. The amount of time taken for each step of the imaging process is drawn from a triangular distribution and uses the means and standard deviations listed in Table 1. For each imaging room, we record the time spent without a patient and the time spent with a patient waiting to transfer to a gantry room.

Each gantry room can service only one patient at a time. Radiation is delivered to each patient from one, two, or three different beam angles. In addition to the time taken to deliver the radiation from each angle, the gantry arm must be repositioned between each angle. The time spent to rotate the gantry arm and treat a beam angle is fixed at 90 and 75 seconds, respectively, as these are purely automated steps. Treatment steps 4 and 10 are performed in the gantry room and their times are drawn from triangular distributions. For each beam angle, the gantry room must determine if the cyclotron is ready to fire. For each room, we record the time spent empty and the time spent waiting for the cyclotron.

The cyclotron provides protons for the gantry rooms. In addition to the 75 seconds a cyclotron spends treating a beam angle, it must wait an additional 45 seconds before servicing a different gantry room. When several rooms have requests for protons, they are serviced first come, first served. For the cyclotron, we record total time spent idle and time spent with one or more outstanding requests for protons.

All runs of our model have one cyclotron. The number of gantry and imaging rooms varies from run to run. Patients arrive at regular intervals that vary from run to run. Imaging rooms were either dedicated to specific gantry rooms or were fully connected to all gantry rooms as shown in Figure 2.
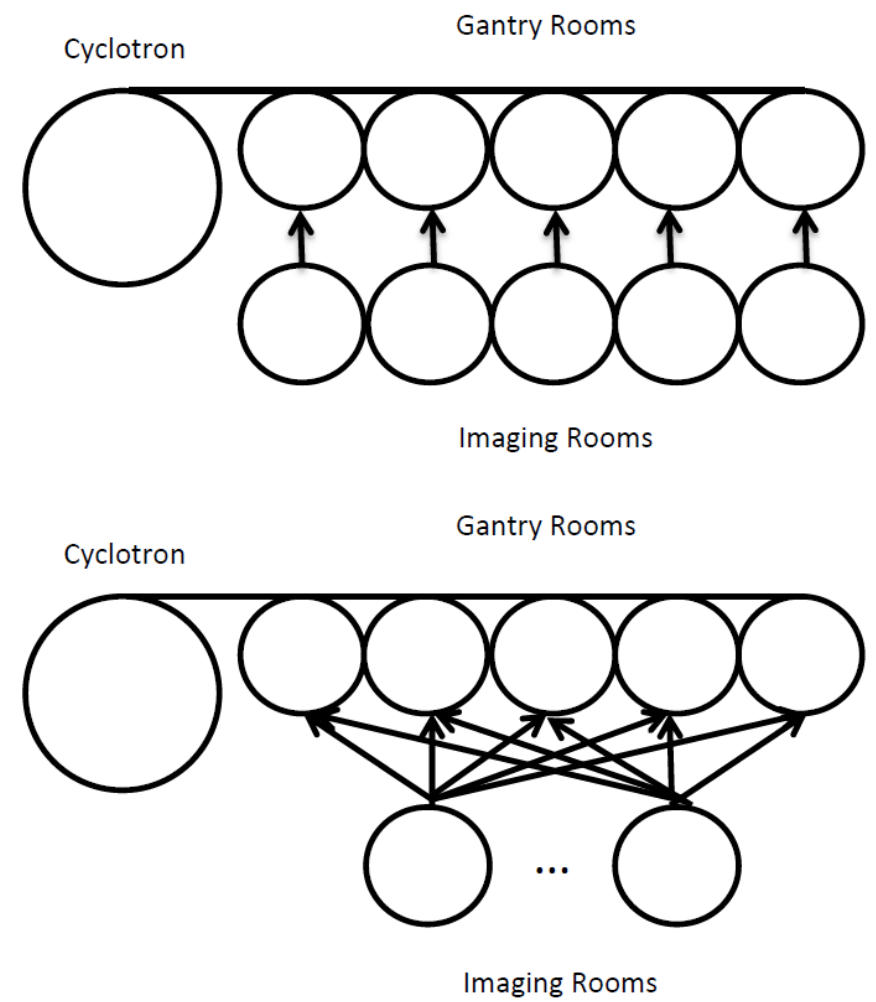

Figure 2: Configurations with dedicated imaging rooms (top) versus shared imaging rooms (bottom). 


\section{SIMULATION RESULTS}

In order to determine the steady-state throughput, we ran simulations for 250,000 ticks (about 340 hours of simulated operation), where the first 50,000 ticks were treated as a burn-in period. The simulations ran with patients arriving whenever an imaging room became available (this could be thought of as an infinite waiting room scenario). The resulting throughput (expressed in patients per hour) provides an upper bound on throughput for one to seven gantry rooms because there is always a patient ready to enter the gantry room. In Figure 3, we see a $26 \%$ increase in throughput by adding a fourth room, another $14 \%$ for a fifth room, but only $6 \%$ for a sixth room. With six rooms, the cyclotron is idle less than $2 \%$ of the time. Therefore, additional rooms can do little to increase throughput and only result in longer patient wait times in the gantry room.
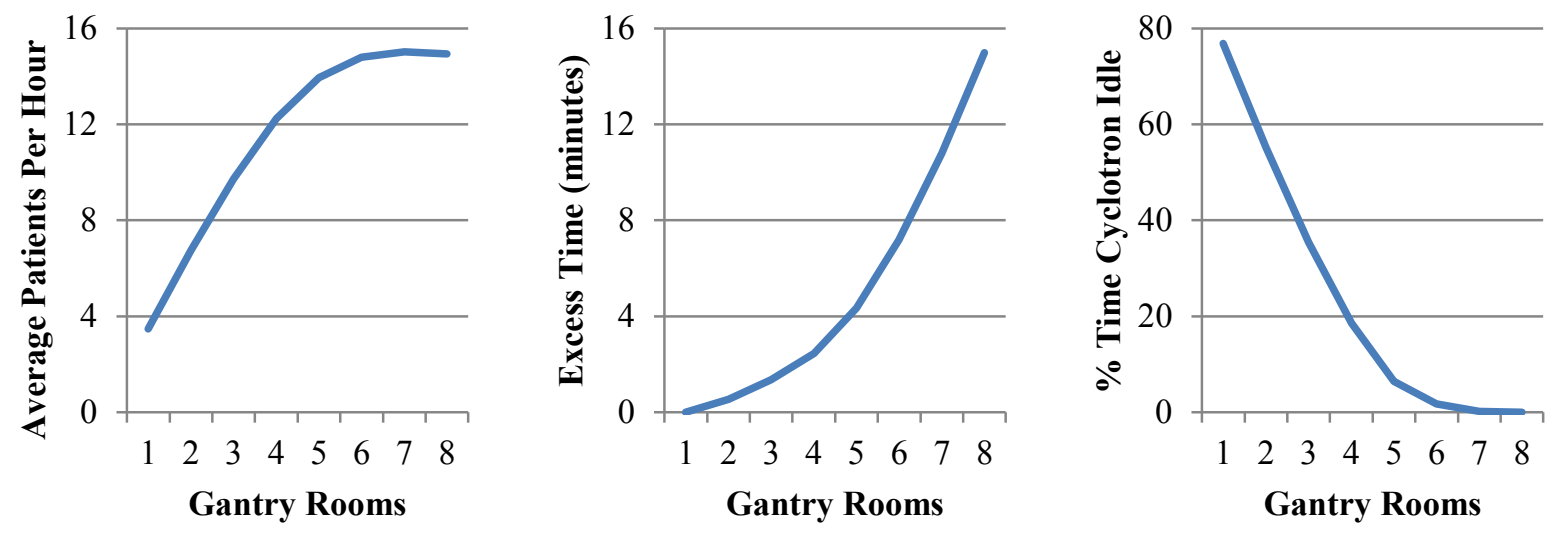

Figure 3: Average patients per hour, excess time in a gantry room (minutes), and percent of time that the cyclotron is idle as a function of the number of gantry rooms for a one cyclotron system. In this system, there is always a patient ready to enter an available gantry room.

The average excess time spent in the gantry room is an aggregate measure. We need to look at the distribution of wait times. From Figure 3, for four gantry rooms, there is an average of 2.46 minutes of excess time spent in the gantry room at maximum capacity. In Figure 4, we see that, under these same conditions, many patients still spend more than three minutes waiting for the cyclotron in the gantry room. As the number of gantry rooms increases, both the mean and variance of wait time increase. The increased variance makes it difficult to bring maximum wait time below three minutes, since the maximum wait time grows faster than average wait time (see Figure 4).

The remaining experiments are performed on 100 days of simulated facility performance. Including daily startup effects is more realistic than using only steady-state behavior. In Table 2, we see that, by decreasing the arrival rate (given by patients per hour), we can reduce the wait times of patients. For example, by increasing the time between patient arrivals in a four gantry room and five imaging room system from 300 seconds (12.00 patients per hour) to 320 seconds (11.25 patients per hour), we reduce the average total wait time by over 55\%. In Figure 5, we see that, even when operating below maximum throughput and having an average wait time of 2.72 minutes, $47 \%$ of the patients have wait times longer than three minutes in the gantry room. 
Price, Golden, Wasil, and Zhang

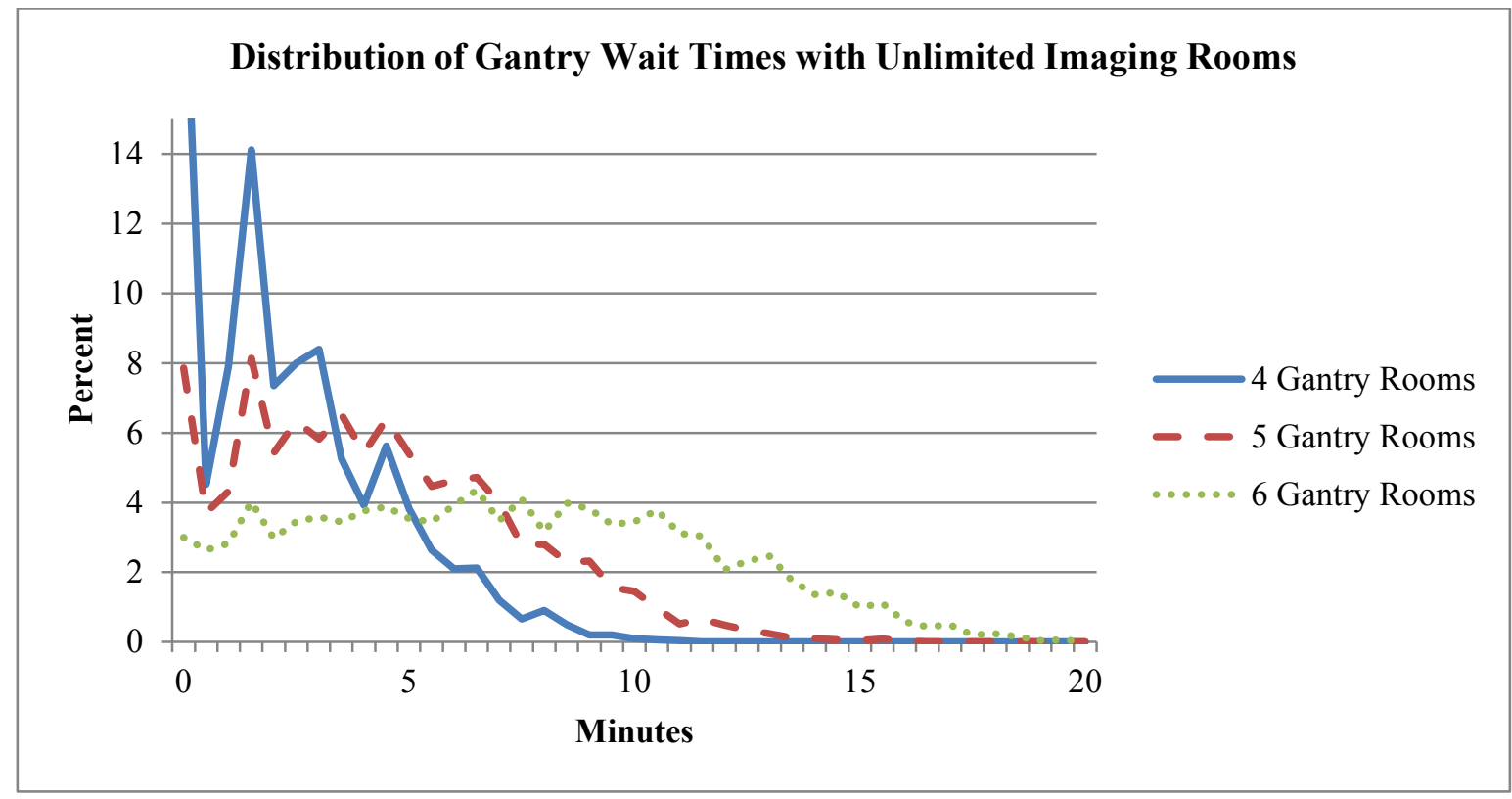

Figure 4: Percentage of patients experiencing various wait times for 10,000 patients.

Table 2: The average wait time by room for six combinations of arrival rates, gantry rooms, and imaging rooms for 100 simulated days of operation.

\begin{tabular}{|c|c|c|c|c|c|c|c|}
\hline \multicolumn{2}{|c|}{ Number of Rooms } & \multicolumn{2}{c|}{ Number of Patients } & \multicolumn{4}{c|}{ Time Spent (minutes) } \\
\hline Gantry & Imaging & Per Hour & Per Day & Waiting & Imaging & Gantry & Total \\
\hline 4 & 4 & 11.25 & 157.50 & 0.51 & 1.33 & 1.87 & 3.71 \\
\hline 4 & 5 & 11.25 & 157.50 & 0.03 & 1.42 & 1.83 & 3.28 \\
\hline 4 & 5 & 12.00 & 168.00 & 1.39 & 3.78 & 2.21 & 7.38 \\
\hline 5 & 5 & 12.00 & 168.00 & 0.00 & 0.40 & 2.63 & 3.03 \\
\hline 5 & 5 & 13.33 & 186.67 & 0.68 & 1.72 & 3.33 & 5.73 \\
\hline
\end{tabular}

There are long wait times in the gantry room; in contrast, $80 \%$ of patients spend no time waiting in the waiting room, $56 \%$ spend no time waiting in the imaging room, and $1.2 \%$ spend no time waiting in the gantry room. The multi-modal nature of the time spent in the gantry room (see Figure 5) is a result of the different treatment plans (one, two, or three beam angles) for patients. We constructed Figure 5 with patients arriving randomly and not taking into account the number of beam angles in the treatment plan. When scheduling patients, it would be important to distribute patients of each type throughout the day, because more beam angles means a longer time in the gantry room on average. For five imaging and gantry rooms, by simply alternating among the three plan types, we reduce the average total wait time by over two minutes and the time spent in the gantry room by 37 seconds. The percent of patients spending more than three minutes in the gantry room falls from $47 \%$ to $39 \%$. The multi-modal nature of the distribution has not changed in Figure 6, but the long tail from consecutive three beam plans (which take more time in the gantry room) now falls off much more quickly. 
Price, Golden, Wasil, and Zhang

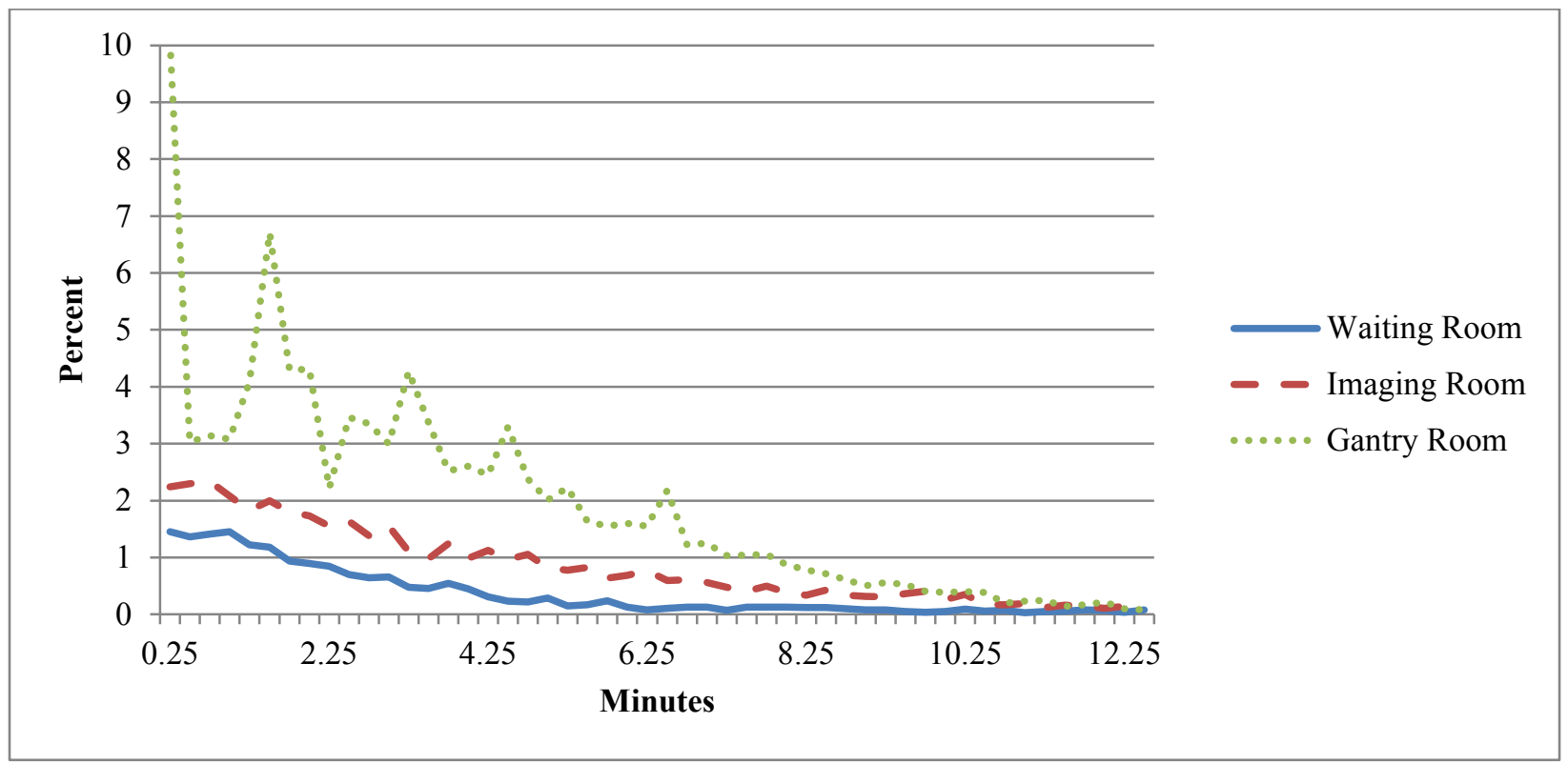

Figure 5: Percent of patients experiencing wait times for a system with five gantry rooms and five imaging rooms using an arrival rate of 13.33 patients per hour (given in Table 2) for 100 simulated days.

It is important to determine the sensitivity of waiting times to real-world factors such as patient tardiness and absenteeism. Both of these factors play important roles in scheduling outpatient procedures (Liu, Ziya, and Kulkarni 2010). The arrival times for patients not staying at the facility are modeled by a symmetric triangular distribution centered on the patient's scheduled arrival time and is 18 minutes in either direction. The 18-minute triangular distribution is an approximation of the fitted distribution found in the empirical study by Liu, Ziya, and Kulkarni (2010). Absenteeism rates are set at 5\% for patients not staying at the facility. Half of the patients are estimated to stay at the facility and thus would be far less likely to be absent or tardy.

In Figure 7, we see that the distribution of waiting times look very similar. Despite seeing $2.5 \%$ fewer patients due to absenteeism, the wait times are longer in each part of the system when patient tardiness is included in the model. There is a $10 \%$ increase in waiting time in the gantry room and a $27 \%$ increase in the total time spent waiting in the system. To address the problem of patient tardiness, we note that patients staying at the treatment center will have greater flexibility in scheduling their treatment time. Patients not staying at the treatment center could be scheduled first, allowing them to choose times when they would be less likely to arrive late. Given that many patients actually arrive early for their appointments in outpatient settings (Liu, Ziya, and Kulkarni 2010), a queue might be created based on patient arrival time instead of patient appointment time. 
Price, Golden, Wasil, and Zhang

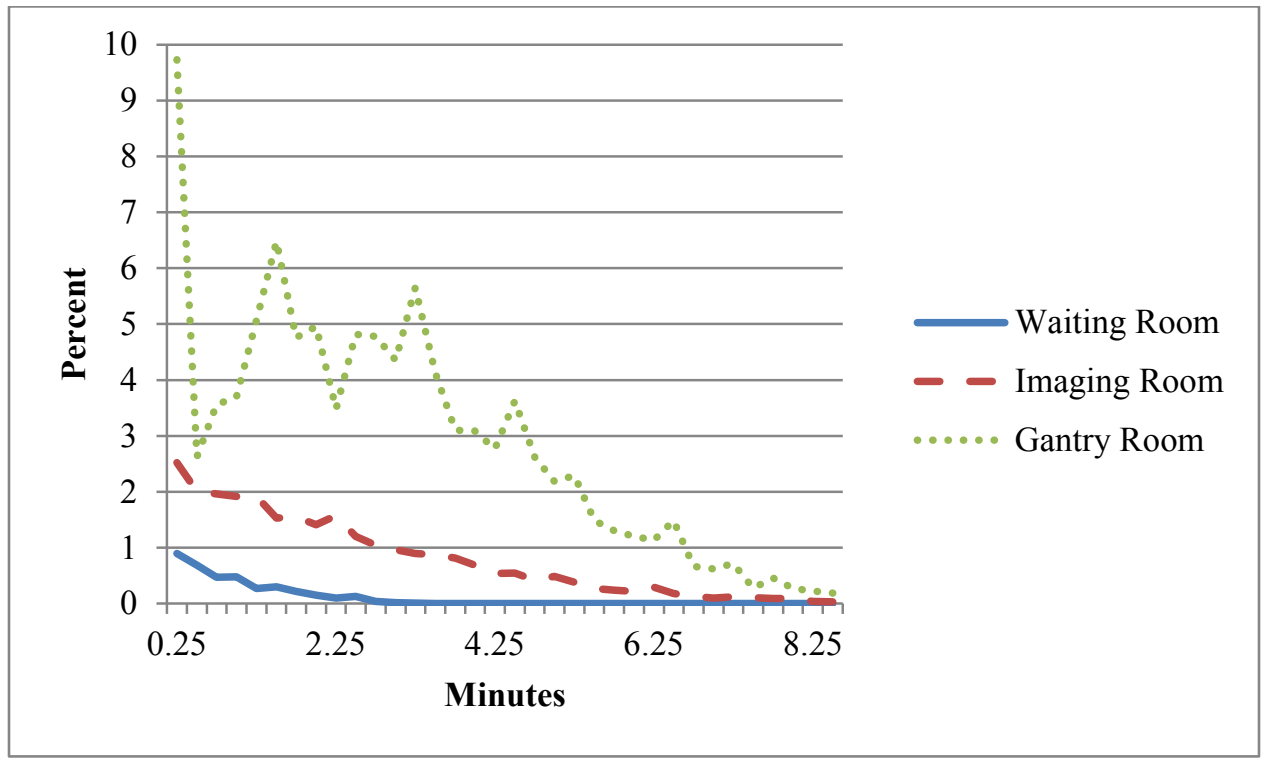

Figure 6: Percent of patients experiencing wait times for a system with five gantry rooms and five imaging rooms with an arrival rate of 13.33 patients per hour for 100 simulated days of patients with alternating treatment plans.

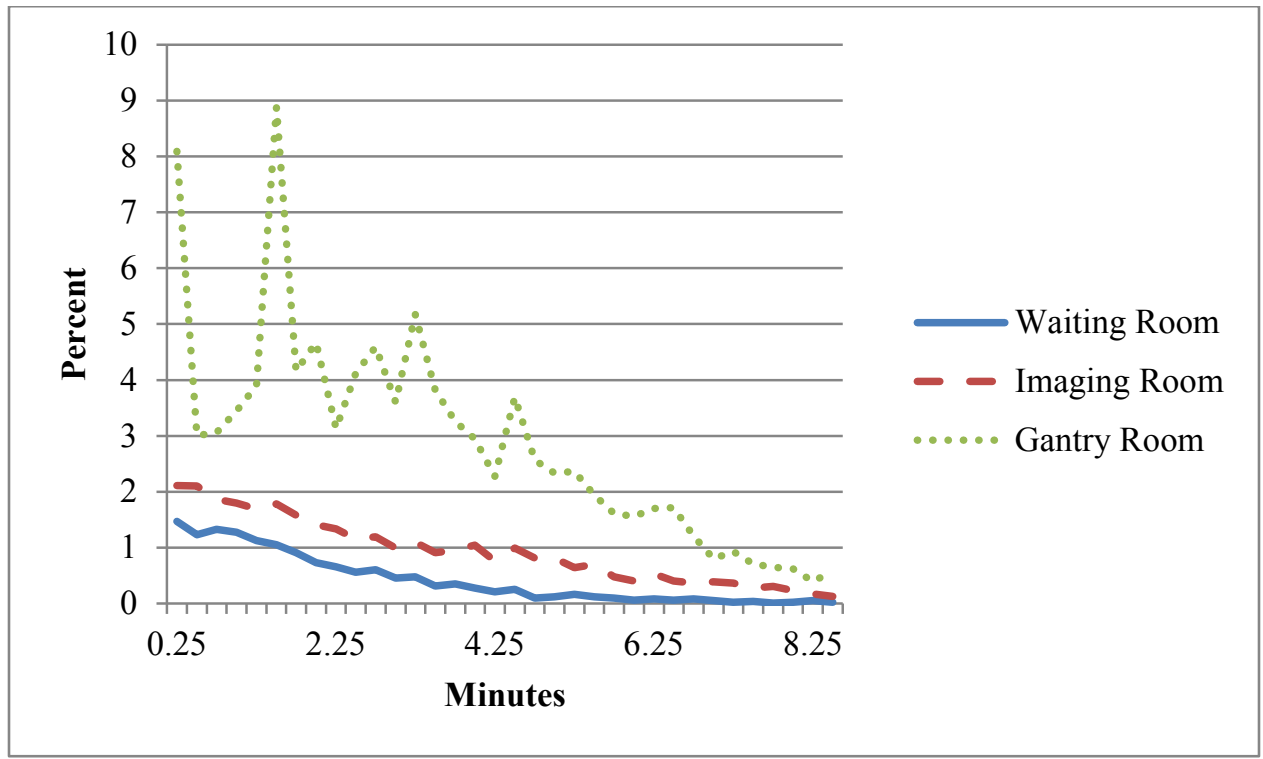

Figure 7: Percent of patients experiencing wait times for a system with five gantry rooms and five imaging rooms with an arrival rate of 13.33 patients per hour for 100 simulated days of patients with alternating treatment plans and late arrivals.

\section{EXTENSIONS TO OUR MODEL}

The time to move between an imaging room and a gantry room is treated the same in our model regardless of which imaging room the patient is moving from and which gantry room the patient is moving to. In remote positioning systems with a single gantry room, the imaging room is directly adjacent to the gan- 
try room. In multi-room complexes, the travel time between an imaging room and a gantry room would vary based on location and hallway capacity. This might lead to a more complicated choice of available gantry rooms based on travel times and the time until patients in the system are finished imaging. We need to ensure that patients in transit would not run into one another, as automated systems are single tracked.

As PT is a developing treatment, best practices are still being determined and are occasionally subject to change. Fava et al. (2012) examined the effects of advances, such as faster imaging and setup correction systems and arc techniques (a delivery technique that would reduce the number of beam angles and the total time spent in the gantry room), on the throughput of PT centers. These advances can be modeled by changing the distributions for the time a patient spends in the various stages of the treatment process.

\section{CONCLUSIONS}

While there are many considerations to take into account when designing the remote positioning system between imaging rooms and gantry rooms, it is important to examine the marginal gain from adding an additional imaging room. The expense of adding a fifth imaging room allowing for an extra 2.1 patients per hour is easier to justify than the sixth room at a marginal increase of 0.8 patients per hour. The complexity of completely connecting all imaging and gantry rooms might not be justified with only a $1 \%$ improvement with five imaging rooms and at most a 5\% improvement with three imaging rooms.

\section{ACKNOWLEDGMENT}

We thank Sean Barnes for his helpful comments on an earlier draft of this paper.

\section{REFERENCES}

Alexopoulos, C., D. Goldsman, J. Fontanesi, D. Kopald, and J. R. Wilson. 2008. "Modeling Patient Arrivals in Community Clinics." Omega 36:33-43.

Bolsi, A., A. J. Lomax, E. Pedroni, G. Goitein, and E. Hug. 2008. "Experiences at the Paul Scherrer Institute with a Remote Patient Positioning Procedure for High-throughput Proton Radiation Therapy." International Journal of Radiation Oncology* Biology* Physics 71:1581-1590.

Fava, G., L. Widesott, F. Fellin, M. Amichetti, V. Viesi, A. J. Lomax, L. Lerderer, E. B. Hug, C. Fiorino, G. Salvadori, N. Di Muzio, and M. Schwarz. 2012. "In-gantry or Remote Patient Positioning? Monte Carlo Simulations for Proton Therapy Centers of Different Sizes." Radiotherapy and Oncology 103:18-24.

Liu, N., S. Ziya, and V. G. Kulkarni. 2010. "Dynamic Scheduling of Outpatient Appointments under Patient No-shows and Cancellations." Manufacturing \& Service Operations Management 12:347-364.

Lundkvist, J., M. Ekman, S. R. Ericsson, B. Jönsson, and B. Glimelius. 2005. "Proton Therapy of Cancer: potential Clinical Advantages and Cost-effectiveness." Acta Oncologica 44:850-861.

National Association for Proton Therapy. 2013. Accessed March 8. http://www.proton-therapy.org/.

Roylance, F. 2010. "University of Maryland to Build Proton Cancer Treatment Center." The Baltimore Sun. $\quad$ http://articles.baltimoresun.com/2010-10-14/health/bs-hs-proton-cancer-20101013_1_protontherapy-cancer-treatment-radiation-therapy [Accessed March 8, 2013].

Schulz-Ertner, D., and H. Tsujii. 2007. "Particle Radiation Therapy Using Proton and Heavier Ion Beams." Journal of Clinical Oncology 25:953-964.

Wilensky, U. 1999. NetLogo. Accessed January 10, 2013. http://ccl.northwestern.edu/netlogo/.

Wroe, A., A. Rosenfeld, and R. Schulte. 2007. "Out-of-field Dose Equivalents Delivered by Proton Therapy of Prostate Cancer." Medical Physics 34:3449-3456. 


\section{AUTHOR BIOGRAPHIES}

STUART PRICE is a doctoral candidate in Operations Management in the Robert H. Smith School of Business at the University of Maryland, College Park. He received his undergraduate degree in physics and mathematics from Hendrix College and a master's degree in applied mathematics from the University of Maryland. His research interests include agent-based modeling and simulation, healthcare applications, and data mining. His email address is sprice@rhsmith.umd.edu.

BRUCE GOLDEN holds the France-Merrick Chair in Management Science in the Robert H. Smith School of Business at the University of Maryland, College Park. He received his undergraduate degree in mathematics from the University of Pennsylvania and his master's and doctoral degrees from the Massachusetts Institute of Technology. He joined the faculty of the University of Maryland Business School in 1976 and served as a Department Chairman from 1980 to 1996. Since 1999, Bruce has served as Editorin-Chief of Networks. Before that, he was Editor-in-Chief of the INFORMS Journal on Computing. His research interests include heuristic search, combinatorial optimization, networks, and healthcare operations management. His email address is bgolden@rhsmith.umd.edu.

EDWARD WASIL is a Professor of Management Science in the Kogod School of Business at American University. He received his doctoral degree from the University of Maryland, College Park. He serves as the feature article editor of the INFORMS Journal on Computing and the associate editor of INFOR: Canadian Journal of Operational Research and Information Processing. His research focuses on network optimization and applications of metaheuristics to optimization problems. He was the American University Scholar/Teacher of the year in 2006. His email address is ewasil@american.edu.

HAO HOWARD ZHANG is an instructor in the Department of Radiation Oncology at the University of Maryland School of Medicine. He received his master's degree in Electrical and Computer Engineering and doctoral degree in Industrial and Systems Engineering from the University of Wisconsin-Madison. His research focuses on applying Operations Research techniques, including optimization, machine learning and simulation, to radiation oncology to improve cancer patient care. His recent work using machine learning techniques to predict pathologic response of locally advanced esophageal cancer with PET/CT features received the Best in Physics Award and the John S. Laughlin Science Council Research Symposium Award from the American Association of Physicists in Medicine. His email address is hzhan001@umaryland.edu. 